\title{
A Review of Techniques for Foetal Electrocardiogram Extraction
}

\author{
Nishant Aggarwal \\ Department of Electronics \\ and Communication \\ Guru Nanak Dev University, \\ Regional Campus, Jalandhar, India
}

\author{
Butta Singh \\ Department of Electronics \\ and Communication \\ Guru Nanak Dev University, \\ Regional Campus, Jalandhar, India
}

\begin{abstract}
Electrocardiogram (ECG) holds high significance in medical diagnostics. Cardiologists consider it as an enduring tool and thus the improvement of the diagnostic quality the signal for various recognitions in different environments become a challenge. The signal acquisition is susceptible to the interference from physiological as well as environmental sources. Foetal ECG provides vital information to the physicist to assist in taking critical decisions especially during labor time. Since the direct contact over foetus is perilous to its health, foetal ECG acquisition becomes a challenging task. There is a time as well as frequency overlap of the stronger maternal ECG over the weak foetal ECG. Thus windowing and simple filtering does not extract these signals. This has encouraged various researchers to dwell deep into innovating such filtering techniques to make the acquired signal qualify for discrete diagnostics. This work focuses on the various algorithms proposed for the foetal extraction in terms of their capabilities and performances.
\end{abstract}

\section{Keywords}

Adaptive algorithm, ECG, foetal, non-adaptive algorithm, non-invasive

\section{INTRODUCTION}

The ECG is one of the oldest and most enduring tools used by cardiologists. The principle behind the ECG consists of the electrical activity that is transmitted throughout the body when the heart is depolarized in order to trigger its contraction followed by repolarisation that results in its expansion. The electrical potentials so generated are measured using contact electrodes on the body surface to get ECG in the form of waveform as shown in the Figure.1.

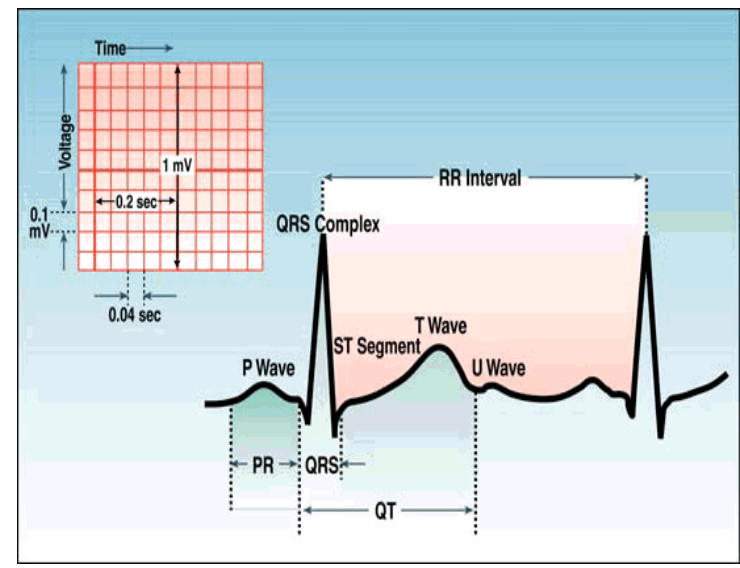

Figure 1: Representation of ECG signal
The extraction of foetal electrocardiogram (fECG) signal has always been a challenging task. The physiologists and engineers have dwelt deep into this emerging field of biomedical signal detection and processing. The foetal heart activity is easily monitored using the fECG. The maturity of the foetus, its development, heart rate, monitoring of foetal distress can be examined using the fECG. Thus it becomes an advantageous tool to parametrically analyze the heart activity of the foetus and to detect neonatal diseases.

Mostly, heart rate is the only information that is being used to monitor the foetus .In order to analyze clinically the heart rate and the allied variations in a wider manner, fECG analysis is needed. But the analysis is generally not undertaken using the morphology of the fECG for the anomalies in heart for several reasons. One of the main reasons is the unavailability of accurate fECG measurement using the available techniques. As compared to prevalent techniques like auscultation and sonography, considering the clinical perspective it is found out that the ECG provides much more information. For the fECG acquisition, two methods are used[1], non invasive methods(maternal abdomen skin electrode) and the invasive (foetal scalp electrode) as shown in Figure 2 and Figure 3 respectively.

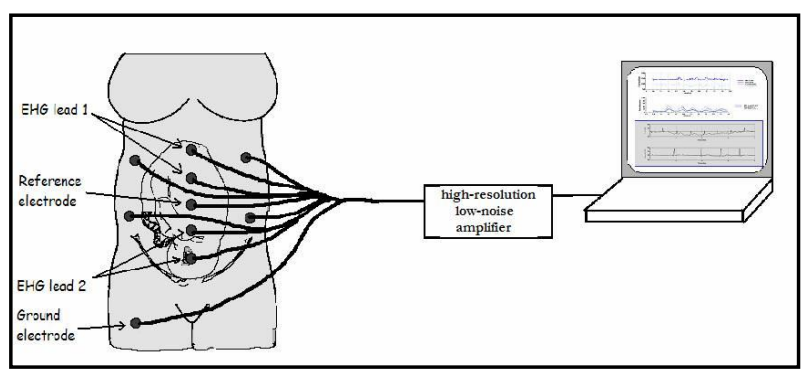

Figure 2: Representation of non invasive fECG acquisition

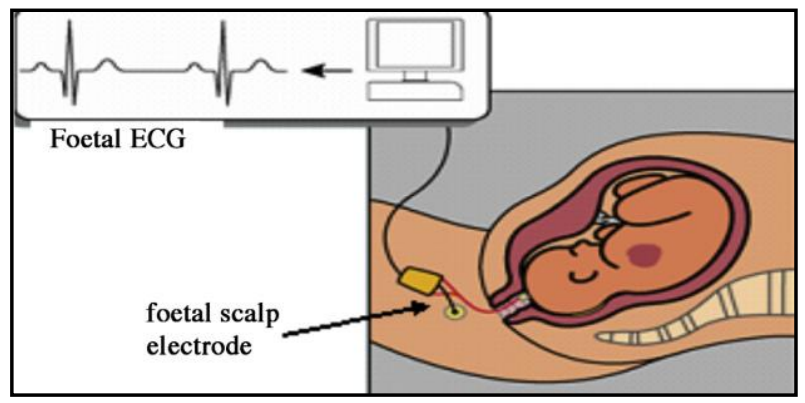

Figure 3: Representation of invasive fECG acquisition[1] 
In the foetal scalp electrode method, the scalp electrodes are placed on the head of the foetus after passing through the womb. This can be a harmful procedure since the electrodes passing through the womb can either rip it or can cause infection and bloodshed. Moreover it can impart undue pressure on the foetus' head. Thus this method is perilous to foetus as well as mother. On the other hand, maternal abdomen skin electrode is non-invasive and thus convenient. Moreover this method can also be used during labor also, which is not the case with the invasive method. Establishing a direct connection with the foetus becomes a challenging task because of the risk involved to the life of foetus while acquiring the ECG. Apart from the risk involved to the foetus, the abdominal measurements contain other physiological and electrical interferences. These interferences can be due to the potentials generated in processes of respiration, maternal muscle and heart activity, foetal heart activity, maternal gastro activity and the maternal ECG interference. There is an overlap in time as well frequency of the strong maternal electrocardiogram (mECG) and the weak fECG in the recordings. Due to this it becomes a tricky task to separate these signals. The recording of the fECG is done from the body of the mother and there is no direct contact to the foetus. The fECG signal is extracted from a signal which is recorded by placing electrodes on the mother's abdomen. This technique has greater vision for foetal well-being and more importantly, long-term monitoring of the foetal heart rate (FHR) can be achieved using innumerable signal-processing techniques. It is completely non-invasive, low power consumption, and can be used over continuous periods of time. Recorded abdominal signal (aECG) contains three types of signals: Maternal ECG signal, foetal ECG signal and interference signal. Some of the important noise sources are muscular (EMG-electromyogram) interference, base line wandering interference, electrodes interference, power line interference, background noise and other interferences. All these interferences have to be eliminated for acquiring a good fECG signal with highest possible fidelity. However, accurate non-invasive elicitation of fECG signal is more challenging, because of two main hindrances. Firstly, the foetus cannot be involved directly for fECG signal. That is no direct contact can be made. Secondly, fECG signal is a very weak signal and superimposed with a strong mECG of mother and other interferences like; power line interference, baseline wander, background noise and other interferences. The foetal signal overlaps with the undesired signals in time, space, frequency, and feature domains. So acquisition of fECG signal becomes more challenging. Therefore methods that only work in one definite sphere are not capable to entirely detach the foetal cardiac signals. In order to develop efficient fECG extraction techniques, various researchers have put in efforts in biomedical engineering and signal processing over many years to help physiologists provide clear ECG. This work analyses such non invasive extraction algorithms proposed. These algorithms can be adaptive and non adaptive depending on capability of each to adaptively cancel the noise or not.

\section{LITERATURE SURVEY \\ 2.1 Non adaptive methods}

The technique of non adaptive methods is the elimination of undesired signals to yield the fECG signal without system adaptation. In other words, the system cannot adjust to the existing circumstances, and simply do the job what it is intended for. These methodologies include wavelet transform based techniques, correlation methods, subtraction methodologies, finite impulse response (FIR) and infinite impulse response (IIR) filtering, wiener filtering and fixed filtering methods such as low pass filtering (LPF) and high pass filtering (HPF).

\subsubsection{Wavelet Analysis method}

Multivariate empirical mode decomposition (MEMD) [2] technique finds application in implementing the methodology of wavelet analysis. In this method the foetal RR time series is extracted using this MEMD technique. The signal is broken into groups of intrinsic mode functions (IMFs) by the MEMD. On the basis of comparison of similarly indexed IMFs, obtained through the MEMD technique, the noisier abdomen ECG channels is eliminated. Thereafter, denoising of the remaining abdomen ECG channels is done. Even after denoising, this noise free abdomen ECG contains some unwanted mother QRS complexes which are hence removed. Eventually, the detection of the foetal R-peaks is done through the wavelet based approach. In [3], a demonstration of the complex continuous wavelet transform (CCWT) for the detection of the QRS wave is done. Based on CCWT, it uses the modulus of the coefficients for the cardiac cycle detection. The methodology consists of four stages, signal averaging, maternal QRS detection, candidate foetal QRS detection and foetal heart rate detection. The dataset used is from the abdominal recordings and also from the three channels of raw ECG data obtained from subjects that have gestation period between 20 and 41 weeks. The sensitivity and the positive predictive accuracy in foetal R-wave detection were $99.4 \%$ and $99.5 \%$. The technique is computationally fast for a good performance. In [4],another wavelet based method to detect foetal heart sound signals shows hoe to denoise and reconstruct the signal for an improved signal to noise ratio. The fECG signal is studied from the abdomen ECG data. The data so obtained is investigated in the time domain using WignerVille distribution. The signal being used in the time domain makes the method computationally easier to examine. Further, [5] uses a spline wavelet method to detect the maternal ECG edges. Local maxima for positive parts and local minima for negative parts are employed to determine the crucial points using the negative threshold. The advantage of this method is that fECG signal estimation can be done using only one channel and also that the $\mathrm{P}$ and $\mathrm{T}$ waveforms can be extracted easily. Disadvantage of this method lies in the difficulty and lack of accuracy in determining the threshold for removing maternal ECG. But, the results show that the computational time is $2 \mathrm{~s}$ which makes it a faster method.

\subsubsection{Blind source separation method}

The BSS (Blind source separation) algorithms use the property of sparseness [6] or statistics of high-order[7,8,9] for detection of signal amidst different noises. There is statistical independence of the sources and need of few assumptions, which makes the problem detection in a completely blind context. On the other hand, the second-order statistics (SOS) $[10,11,12]$ operate in a semi-blind setting. The derivation here usually requires some more assumptions that are made on the source signal. These assumptions can be cyclostationarity in the signal, time correlations in the stationary signals or the statistical nonstationarity of the sources. [13] enlightens another algorithm for FECG extraction when additive noise is present. The updating rule used here makes the objective function to decrease monotonically and then extract the desired signals when the objective function reaches a minima. The wavelet analysis method and BSS method can be 
combined to form another technique [14] to separate the maternal and foetal signals from combined ECG. A Blind adaptive-filtering technique that overcomes the limitations of the basic BSS methods uses ICA to extract fECG as shown in [15]. The reconstruction is done using the wavelet method.

A simple and fast algorithm that is based on independent component analysis to estimate the foetal electrocardiogram (FECG) is shown in [16]. The main advantage lies in substitution of the computationally demanding calculations with a much simpler procedure. The steps include dimensionality reduction followed by a post processing stage that is used to enhance the FECG signal. The advantage lies in the post processing stage being computationally light.

\subsubsection{Subtraction method}

The aECG and mECG signals are aligned in such a way that the mECG signal can be subtracted from the aECG signal. The resulting signal is the fECG signal with noise. These noises then need to be filtered out. Event synchronous interference canceller (ESC)[17] demonstrates the removal of mECG from signal recorded abdominally during pregnancy. Advantage lies in the use of compensation method to process the abdominally recorded signal. The amplitude as well as the shape of the signal can be easily restored using the basic compensation concept [18]. The ESC algorithm is basically a subtraction algorithm and is modest with respect to computational load.

Further, [19] proposes a subtraction method in which the thoracic mECG is subtracted from an aECG signal which is a combination $\mathrm{mECG}$ and fECG signal. Synthetic and real time data are used for experiments. The major difficulty of this technique is the measurement of the thoracic $\mathrm{mECG}$ rarely matching the scale of the mECG present in the aECG signal. Consequently, a pure fECG is hardly ever yielded when the signals are being subtracted.

[20] provides another procedure using subtraction methodology where power line interference elimination from ECG signals is achieved. Different types of notch filters can be used for its implementation. The subtraction procedure does not seem to affect the frequency components of the ECG signal around the frequency of interference. In order to remove the interference components from the signal, digital filtering is used on the linear segments of the signal. These interference components are subtracted from the signal wherever non-linear segments are detected.

\subsubsection{Correlation based method}

An averaged mECGsignal is obtained using a suitable correlation function from the two signals via mECG and aECG. This correlation function is then subtracted from the aECG to yield the desired fECG signal.

Although correlation techniques are not generally effective in the detection of non-stationary signals like ECG, still some researchers provides ways to achieve the extraction using this method.[21] shows a correlation technique for detecting the presence of a foetal heart signal in an aECG signal corrupted by noise. The method includes auto correlation as well as cross correlation techniques. Synthetic and real time data are used for experimentation. Results show that the extracted fECG is effective but not efficient enough. This is the expected result of correlation techniques for non-stationary signals. The combining of non-Gaussianity and timecorrelation of the source signals for fECG extraction can be helpful in overcoming these limitations. A promising approach [22] is the blind source extraction where an objective function for extraction of temporally correlated sources is developed. The objective function is based on the autocorrelations of source signals and the non-Gaussianity. The concept used is the minimization of the objective function and mean squared error objective function is used in this case . The algorithm thus extracts the fECG and is very robust in the estimation of the time delay error.

\subsection{Adaptive methods}

Adaptive filters are filters that have self-designing algorithms implemented in it. The filter learns the initial input statistics and continuously track these for time variations. Thus the estimation can be done for the deterministic signals. The noise that is uncorrelated with the deterministic signal can be thus eliminated. Recursive algorithm are used to design these self designing adaptive filters and find application there is lack of knowledge a priori. Figure.4 shows an Adaptive Filter Structure. Here the input signal obtained from sensors is $d(n)$. It contains the desired signal d'(n) as well as the undesired signal $u(n)$. $u(n)$ can be considered as the noise signal distorting the measured signal. Now, if the noise signal $u(n)$ is known a priori, desired signal d'(n) can be extracted by subtracting noise signal $u(n)$ from input signal $d(n)$ which is corrupted with noise. It is difficult to obtain the entire noise signal, thus an estimated noise signal $\mathrm{y}(\mathrm{n})$ is used. Filters are

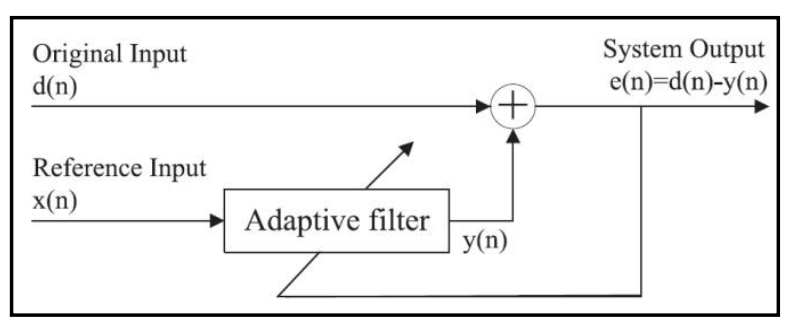

Figure 4:Adaptive filter structure

used to estimate the noise signal y (n), There is a linear relation between the measurable noise source $x(n)$ and the noise signal $y(n)$. The estimated signal $y(n)$ is then used to obtain the difference signal e(n) using which the estimation of the desired signal d'(n) is done. The closeness of estimated noise signal $y(n)$ with the real noise signal $u(n)$ determines the

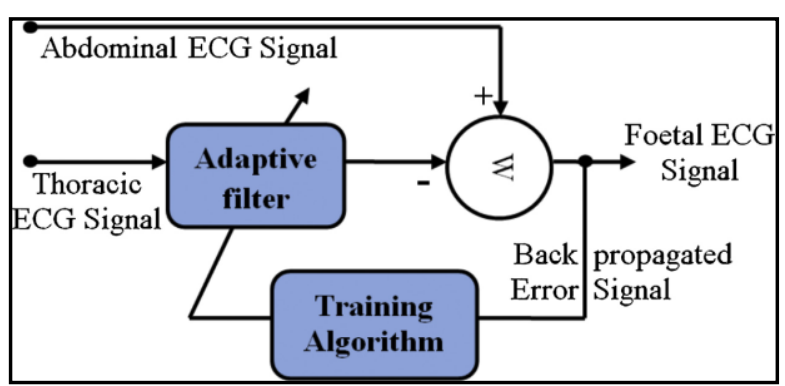

Figure 5: Adaptive noise filter for foetal ECG extraction

value of the desired signal. Adaptive filtering implements noise cancellation theory. Adaptive filter design[1] can be classified into two parts, digital filter and adaptive algorithm implementation. Function of adaptive algorithm is to generate proper filter coefficient. General digital filters use fixed filter coefficients, This is not the case with adaptive filter, Here a 
change in filter coefficients in considered on the basis of input and output signal characteristics as well as the environmental changes. A typical adaptive noise cancellation (ANC) system shown in Figure 5 would be the best example of an adaptive elicitation technique. It consists of two signals, the noisy abdominal ECG signal is the primary input and a thoracic ECG signal is used as the reference input. The abdominal signal consists of mainly maternal heartbeat which dominates the foetal heart beat and other residual noises. The thoracic signal consists of the actual maternal heart beat with additive noise. The Finite Impulse Response Filter weights will be updated by the training algorithm based on the back propagated error signal, which is actually the desired fECG signal. Maternal heart beat is considered to be noise for elimination. The adaptive filter produces a signal which is approximately the noise. This signal is subtracted with the aECG signal so that the error signal that is back propagated to the training algorithm is the foetal heart signal with some noise. The training algorithm updates the filter coefficients. The noises are adaptively filtered out to yield the desired foetal ECG signal.

\subsubsection{LMS Adaptive filtering}

Adaptive filtering is generally implemented using the basic filtering scheme in combination with an adaptive filtering scheme. In [23], a basic ECG extraction method used is the wavelet analysis. The least mean square (LMS) filter is used adaptively and algorithm so implemented makes it an adaptive noise cancellation scheme. Also used is the spatially selective noise filtration (SSNF) algorithm to further eliminate the environmental effects adaptively. The stationary wavelet transform (SWT) is used to process the signals and the generation of wavelet coefficients at each scale are done. These coefficients are then processed using the LMS filter. The input of the LMS adaptive filtering system is the coefficient of the abdominal signal, and the reference input is the coefficient of the thoracic signal. Then computation of wavelet coefficients is done using the correlations of the processed signals. The threshold is determined and eventually the noise components are removed by the SSNF algorithm. Also signed LMS that is normalized at each regression can also be used for the noise elimination as shown in [24].

Thus these techniques use a basic LMS filtering scheme to adaptively filter the noises to extract the foetal ECG.

\subsubsection{Neural networks}

Neural networks benchmarks with the classical adaptive methods such as the least mean squares algorithms. The compromise between complexity of the model and with its outcomes is reached by the FIR neural networks. Selection of a model and the introduction of an advanced neural model determine the methodology that can be used for this purpose. Dynamically capable recovery model is needed to implement highly non-linear systems. Gamma neural networks and finite impulse response (FIR) [25] are also inculcated in the adaptive noise cancellation (ANC) schemes to provide improved performances. Other implementations can be done using hardware prototyping[26] , radial basis[27] functioning or using basic FIR structure[28] to link with the neural networking model and thus obtain enhanced results. It is required to identify the nonlinear relationship and then to align the signals with each other, for this purpose adaptive neuro-fuzzy inference systems (ANFIS)[29] can be implemented that helps in extraction of fECG with least additive noise. ANFIS network helps in aligning the mECG signal in the abdominal ECG signal with the mother ECG signal component. The fECG embedded in the maternal QRS complex can be easily extracted out using this method. In [30], an adaptive neuro fuzzy logic technique for the elicitation of fECG signal by eliminating the mECG signal from the aECG signal is proposed. The experiments are carried out with the simulated signals and real ECG signals are used for elicitation. Adaptive neuro fuzzy inference system (ANFIS) cancels out the interference and gives better performance even for a high complex signal. In [31], a fuzzybased approach for the elicitation of foetal heart rate (FHR) by detecting the QRS complex in the fECG signal is developed. The designed QRS detection algorithm uses a fuzzy decision technique to classify maternal and foetal ECG from single lead maternal abdominal recordings.

\subsubsection{Phase space Adaptive filtering}

The technique of phase space signal filtering [32][33] can be used to adaptively filter the foetal ECG as well. The reconstruction of the phase space trajectory with thoracic ECG signal and abdominal ECG taken from a pregnant women can be achieved using an adaptive filtering[34],where there is a time variant and non-linear relation between the maternal ECG signal and thoracic ECG signal. Adaptive local modeling in phase space is used to estimate the maternal ECG component which is present in the abdominal ECG signal. In order to derive an estimated foetal ECG this signal is then subtracted from the abdominal ECG signal. Then local modeling is carried out on the spatially adjacent points related to each point in phase space. Thus utilization of the information contained in the geometric representation of the periodic ECG signal, as well as the local relationship represented in phase space is done to estimate the relationship in more easier and accurate manner. This method is robust and there is fair amount fidelity achieved while restoring the foetal ECG.

\subsubsection{Adaptive comb filtering}

There can be temporal variations in the fundamental frequency and some quasi-periodic components in the input signal. Such a signal qualifies for the estimation using adaptive comb filter (ACF). [35] describes one such robust and simple algorithm to extract foetal electrocardiogram from abdominal signal using ACF. This advances the comb filter sampling [36]by the same authors. The basic comb filtering technique described in [37][38] where adaptive adjustment to the temporal variations in fundamental frequency is achieved. It allows some harmonics of the signal to pass but filters out spectral components between the harmonics in the frequency region. The fECG estimation algorithm using ACF is composed of two cascaded parts. The first estimates the maternal ECG (mECG) component in the abdominal signal and subtracts it, and the second enhances the fECG in the residual signal. These two sets of operations form the basis of operation in this two-fold filtering problem based on ACF with the fundamental frequency of MECG and fECG respectively. The described ACF algorithm could serve both in mECG estimation stage and fECG enhancement stage according to fundamental frequency of $\mathrm{mECG}$ and $\mathrm{fECG}$ respectively. Thus the maternal $\mathrm{R}$-wave peaks in the abdominal signal and the fetal $\mathrm{R}$-wave peaks in the residual signal should been detected in advanced. 


\subsubsection{Bayesian methodologies}

Bayesian system frameworks are techniques of estimation in which Bayes' rule is utilized to update the probability estimate for a supposition as additional verification. These frameworks evaluate the estimates of the hidden system state over time using mathematical models and observed quantity. Several variants of Bayesian filters are available for solving the problems with non linear signals such as the ECG signal. Bayesian methods for fECG signal extraction can be integrated with PCA technique [39]. The probabilistic model is formulated in a state - space form and the Expectation Maximization algorithm is used to derive the maximum likelihood (ML) values of the parameters. This is then extended to a Bayesian approach. Denoising of signals can also be achieved [40] by implementing the Bayesian filtering framework. Several filtering combinations and optimal filters can be used different noise removals and results perfected in terms of better SNR are achieved. Further, fECG can also be extracted using Bayesian inference with Neural Networks[41]. Here the ECG signal is modeled by generalized Gaussian distribution and back propagation neural network is used to approximate the nonlinear function considering the two signals mECG and aECG. Also, an adaptive Kalman Filter can be used to enhance the SNR of ECG signal[42]. For longterm monitoring tasks, the ECG signal characteristics may change, thus adaptive Kalman filter adapts itself to the noise estimation and matches the filter's output to the new input. The Kalman filtering frameworks are promising approaches for mECG deletion and fECG enrichment. This framework laid the foundation for extensions on more highly developed filters such as the Particle filter. The drawback of Bayesian modeling and Kalman filtering are that it involves mathematical complexity, computational time more and requires a detailed information on the ECG signal dynamics.

Table.1 Comparison of different foetal ECG extraction techniques

\begin{tabular}{|c|c|c|c|}
\hline Author & Methodology & $\begin{array}{l}\text { Computational time } \\
\text { (seconds) }\end{array}$ & Accuracy (\%) \\
\hline Gupta[2](2016) & $\begin{array}{l}\text { Multivirate empirical mode } \\
\text { decomposition }\end{array}$ & 0.01 & 100 \\
\hline Karvounis[3](2004) & $\begin{array}{l}\text { Complex continuous wavelet transform } \\
\text { technique }\end{array}$ & 4 & 98.94 \\
\hline Karvounis[43](2010) & Blind source separation technique & & 94.79 \\
\hline $\operatorname{Azad}[45][2000]$ & Fuzzy approach & & 89 \\
\hline Ungureanu[44](2009) & $\begin{array}{l}\text { Adaptive maternal beat subtraction } \\
\text { technique }\end{array}$ & & 93.7 \\
\hline Shi[22](2007) & Semi-blind source extraction technique & & 99.74 \\
\hline Rahman[24](2011) & $\begin{array}{l}\text { Sign based normalized adaptive filtering } \\
\text { Technique }\end{array}$ & & 81 \\
\hline Assaleh[29](2007) & Adaptive neuro-fuzzy approach & $<0.8$ & \\
\hline Wei[35](2013) & Adaptive comb filtering technique & 0.25 & \\
\hline Vullings[42](2011) & Adaptive kalman filtering technique & & 85 \\
\hline Piere[46](2001) & Matched filtering technique & & 65 \\
\hline Ibrahimy[47](2003) & Statistical analysis & & 89 \\
\hline Martens[48](2007) & Principal component analysis & & 85 \\
\hline Karvounis[49](2007) & $\mathrm{t}-\mathrm{f}$ analysis & & 99.19 \\
\hline Karvounis[50](2009) & 3D phase space analysis & & 95.45 \\
\hline
\end{tabular}

\section{CONCLUSION}

In this paper, elaborate portrayal is done of the diverse techniques used for fECG extraction through elimination of interference and other signal processing techniques. The merits and demerits of the various techniques are analyzed in a detailed manner for possible usage for researchers to derive novel ideas. The aim of this work is to provide concise information about fECG extraction techniques and to reveal the diverse issues related to the signal extraction and methodologies to analyze the signal for efficient fECG signal 
extraction. The techniques are discussed along with their advantages and drawbacks. This amendment clearly points out the various techniques implemented during the past years, so that the areas of concerns can be improved and accurate signal processing methodologies can be designed for fECG signal extraction and applied real time during medical diagnosis. The promising technology and latest signal processing methodologies do have the potential for a positive impact on the issue.

\section{REFERENCES}

[1] Jagannath, D. J., and Selvakumar, A. I. 2014. Issues and research on foetal electrocardiogram signal elicitation, Biomedical Signal Processing and Control. 10, 224-244.

[2] Gupta, P., Sharma,K.K., and Joshi, S.D. 2016. Fetal heart rate extraction from abdominal electrocardiograms through multivariate empirical mode decomposition. Computers in Biology and Medicine. 68,121-136.

[3] Karvounis, E.C., Papaloukas, C., Fotiadis, D.I. and Michalis, L.K. 2004. Fetal heart rate extraction from composite maternal ECG using complex continuous wavelet transform, Computer Cardio 1,737-740.

[4] Song, Y., Xie, W., and Chen, J.F. 2006. Passive acoustic maternal abdominal fetal heart rate monitoring using wavelet transform. Computer Cardio,581-584.

[5] Sargolzaei, S., Faez, K., and Sargolzaei,A. 2008. Signal processing based for fetal electrocardiogram elicitation. In Proceedings of the International Conference on Biomedical Engineering and Informatics.

[6] Zibulevsky, M., and Zeevi Y.Y. 2002. Extraction of a source from multichannel data using sparse decomposition. Neurocomputing 49 (1-4),163-173.

[7] Liu, W., and Mandic, D.P. 2006. A normalized kurtosis based blind source extraction from noisy mixture. Signal Process. 86(7), 1580-1585.

[8] Zhang, Z.L., and Yi, Z. 2006. Extraction of a source signal whose kurtosis value lies in a specific range, Neurocomputing 69 (7-9),900-904.

[9] Lathauwer, L., Moor, B., and Vandewalle, J. 2000. Fetal electrocardiogram extraction by blind source subspace separation. IEEE Trans. Biomed. Eng. 47(5),567-572.

[10] Cichocki, A., and Thawonwas, R. 2000. On-line algorithm for blind signal extraction of arbitrarily distributed, but temporally correlated sources using second order statistics. Neural Process. Lett. 12 (1),9198.

[11] Zhang, Z.L., and Yi, Z. 2006. Robust extraction of specific signals with temporal structure. Neurocomputing 69 (7-9), 888-893.

[12] Barros, A.K., and Cichocki, A. 2001. Extraction of specific signals with temporal structure. Neural Comput. 13 (9), 1995-2003.

[13] Yunxia, L., and Zhang, Y. 2008.An algorithm for extracting fetal electrocardiogram. Neurocomputing 71, $1538-1542$

[14] Hasan, M.A. and Reaz,I. 2009. Detection and Processing Techniques of FECG Signal for Fetal Monitoring. Biological Procedures Online. vol.11(1),263-295.
[15] Raj, J., Prabhu, V., Christophera, J., Sugumar, D., and Vanathi, P.T. 2012. Separation Of Maternal And Fetal ECG Signals From The Mixed Source Signal Using FASTICA, International Conference on Communication Technology and System Design 2011 Procedia Engineering 30, 356-363.

[16] Clemente, R.M., Olivares, J.L., Mellado, S.H., and Roman, M.I. 2011.Fast Technique for Noninvasive Fetal ECG Extraction, IEEE transactions on biomedical engineering vol. 58. no. 2.

[17] Mihaela, G., Johannes, U., Bergmansb, W.M., Guid, O.S., Ungureanua, A., and Wolfd, W. 2009. The event synchronous canceller algorithm removes maternal ECG from abdominal signals without affecting the fetal ECG,Computers in Biology and Medicine 39, 562 - 567.

[18] Widrow, B., Glover, J.R., McCool, J.M., Kaunitz, J., Williams, C.S., Hearn, R.H., Zeidler, J.R., Dong, E. and Goodlin, R.C. 1975. Adaptive noise cancelling: principles and applications, Proc. IEEE 63 (12),16921716.

[19] Bergveld,P., and Meijer, W.H.J. 1981.A new Technique for the suppression of the MECG,IEEE Trans. Biomed. Eng. 28 (4),348-354.

[20] Levkov,C., Mihov, G., Ivanov, R., Daskalov, I., Christov, I., and Dotsinsky I. 2005.Removal of powerline interference from the ECG: a review of the subtraction procedure. BioMed. Eng. Online 4 (4),50.

[21] Van,J.H., and Bemmel. 1968. Detection of weak foetal electro-cardiograms by autocorrelation and cross correlation of envelopes. IEEE Trans. Biomed. Eng. BME-15 (1),17-23.

[22] Shi, Z., and Zhang, C. 2007. Semi-blind source extraction for fetal electrocardiogram extraction by combining non-Gaussianity and time-correlation. Neurocomputing 70 (7-9), 1574-1581.

[23] Wu,S., Shen,Y., Zhou,Z., Lin,L., and Zeng,Y. Gao,X. 2013. Research of fetal ECG extraction using wavelet analysis and adaptive filtering. Computers in Biology and Medicine 43, 1622-1627.

[24] Rahman, M.Z.U., Shaik, R.A., and Reddy, R K. 2011. Efficient sign based normalized adaptive filtering techniques for cancelation of artifacts in ECG signals: application to wireless biotelemetry. Signal Process. 91 (2),225-239.

[25] Valls, G.C., Sober, M.M., Rafael, E.S., Benedito, M., Maravilla, J.C., and Martinez, J.G. 2004. Foetal ECG recovery using dynamic neural networks. Artificial Intelligence in Medicine 31, 197-209.

[26] Hasan,M., and Reaz,M. 2012. Hardware prototyping of neural network based fetal electrocardiogram extraction. Meas. Sci. Rev. 12,52-55.

[27] Pu, X.J., Zeng, X.P., Chen, Y.J., Yu, W., Han, L., and Cheng, J. 2009. Fetal electrocardiogram extraction based on radial basis function neural networks, J. Chongqing Univ.32,111-115.

[28] Camps, G., Martínez, M., and Soria,E. 2001.Fetal ECG Extraction using an FIR Neural Network. Computers in Cardiology,249-252. 
[29] Assaleh, K. 2007.Extraction of Fetal Electrocardiogram Using Adaptive Neuro-Fuzzy Inference Systems. IEEE transactions on biomedical engineering3.vol. 54. no. 1.

[30] Vijila, C. K.S., Kanagasabapathy, P. and Johnson, S. 2005. Adaptive neuro fuzzy inference system for elicitation of fECG. Annual IEEE India Conferenceindicon, 224-227.

[31] Azad, K.A.K. 2000. Fetal QRS complex detection from abdominal ECG: a fuzzy approach. In the proceedings of IEEE Nordic Signal Processing Symposium, Kolmarden. Sweden, 275-278.

[32] Neves, Fernandes, J.A.C. and Restivo, F. 2006. Phase space signal filtering. Proc. IEEE ICIT, 1805-1809.

[33] Wu, Ji, Jiang, Q.H., and Tang, L.2011. Disturbance detection location and classification in phase space. IET Gener. Trans. Distrib. 5(2), 257-265.

[34] Wei, Z., Hongxing,L., and Jianchun,C. 2012. Adaptive filtering in phase space for foetal electrocardiogram estimation from an abdominal electrocardiogram signal and a thoracic electrocardiogram signal. IET Signal Processing. Vol. 6, Issue. 3, 171-177.

[35] Wei, Z., Xueyun, W., Jian, Z., and Hongxing, L. 2013. Noninvasive fetal ECG estimation using adaptive comb filter, computer methods and programs in biomedicine $112,125-134$.

[36] Wei, Z., Hongxing, L., Aijun, H., Xinbao, N., and Jianchun,C. 2010. Single-lead fetal electrocardiogram estimation by means of combining R-peak detection, resampling and comb filter.Medical Engineering \& Physics 32 (7),708-719.

[37] Frazier, R.H., Samsam, S., Braida, L.D., and Oppenheim, A.V. 1976.Enhancement of speech by adaptive filtering. In the Proceedings of IEEE Int. Conf. on Acoust. Speech, and Signal Processing,Philadelphia.PA API(12-14),251253.

[38] Lim, J.S., Oppenheim, A.V. and Braida, L.D. 1978. Evaluation of an adaptive comb filtering method for enhancing speech degraded by white noise addition. IEEE Transactions on Acoustics Speech and Signal Processing.ASSP-26 (August, 1978), 354-358.

[39] Oikonomou, V.P. and Fotiadis, D.I. 2005. A Bayesian PCA approach for fetal ecg extraction.In the proceedings of the 3rd European Medical and Biological Engineering Conference EMBEC'05. November 20-25. Prague. Czech Republic. vol. 11. no. 1, 1-5.
[40] Sameni, R., Shamsollahi, M.B., Jutten,C. and Clifford,G.D. 2007. A nonlinear bayesian filtering framework for ECG denoising. In the proceedings of IEEE Trans. Biomed. Eng.

[41] Yin, Y., Ye, M., Ren, D., Zhu, Y., and Yang, C. 2010.FECG extraction using bayesian inference and neural networks approximation. Computat. Inform. Syst. 6, 1769-1778.

[42] Vullings, R., Vries, B. and Bergmans, J.W.M. 2011. An adaptive kalman filter for ECG signal enhancement. IEEE Trans. Biomed. Eng. 58 (4), 1094-1103

[43] Karvounis, E. C., Tsipouras, M. G., Papaloukas, C., Tsalikakis, D. G. and Naka, K. K., Fotiadis,D.I. 2010.A non-invasive methodology for fetal monitoring during pregnancy, Methods Inform. Med. 49 (3), 238-253.

[44] Ungureanu, M., Bergmans, J.W.M., Oei,S.G., and Strungaru, R. 2007. Fetal ECG elicitation during labor using an adaptive maternal beat subtraction technique. Biomedizinische Technik Bio Med. Eng. 52 (1),56-60.

[45] Azad, K.A.K. 2000. Fetal QRS complex detection from abdominal ECG: a fuzzy approach. In the proceedings of IEEE Nord. Signal Process. Symp. Kolmarden .Sweden, 275-278.

[46] Piere, J.F, Crowe, J.A., Hayes-Gill, B.R., Spencer, C.J., Bhogal, K., and James, D.K. 2001. Compact long-term recorder for the trans- abdominal foetal and maternal electrocardiogram. Med. Biol. Eng. Comput. 39.118125 .

[47] Ibrahimy, M.I., Ahmed, F., Ali, M.A.M., and Zahedi, E. 2003. Real-time signal processing for fetal heart rate monitoring. IEEE Trans. Biomed. Eng. 50,258-262.

[48] Martens, S.M.M., Rabotti, C., Mischi, M., and Sluijter, R.J. 2007. A robust fetal ECG detection method for abdominal recordings. Physiol. Meas. 28, 373-388.

[49] Karvounis, E.C., Tsipouras, M.G., Fotiadis, D.I., and Naka, K.K. 2007. An automated methodology for fetal heart rate extraction from the abdominal electrocardiogram. IEEE Trans. Inf. Technol. Biomed. $11,628-638$.

[50] Karvounis, E.C., Tsipouras, M.G., and Fotiadis, D.I. 2009. Detection of fetal heart rate through 3-D phase space analysis from multivariate abdominal recordings. IEEE Trans. Biomed. Eng. 56, 1394-1406. 